\title{
Diversity of Staphylacaccus species isolated from surface waters
}

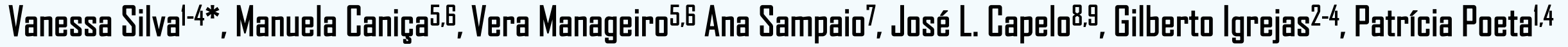 \\ IMicrabiology and Antibiotic Resistance Team (MicraART), Department of Veterinary Sciences, University of Trás-as-Montes and Alta Douro (UTAD), Vila Real, Portugal; \\ 2Department of Genetics and Biotechnology, University of Trás-os-Montes and Alta Douro, Vila Real, Partugal: \\ 3Functional Genomics and Proteamics Unit, University of Trás-as-Montes and Alto Douro (UTAD), Vila Real, Portugal; \\ ${ }^{4}$ Associated Laboratory for Green Chemistry (LAQV-REQUIMTE), University NQVA of Lisboa, Lisboa, Caparica, Portugal; \\ 5National Reference Laboratory of Antibiotic Resistances and Healthcare Associated Infections (NRL-AMR/HAl), Department of Infectious Diseases, National Institute of Health Dr Ricarda Jarge, Av. Padre Cruz, IG49-DIG, Lisbon, Portugal: \\ ${ }^{6}$ Centre for the Studies of Animal Science, Institute of Agrarian and Agri-Food Sciences and Technologies, Oporto University, Oporto, Portugal; \\ ?entre for the Research and Technology of Agro-Enviranmental and Biological Sciences (CITAB), University of Trás-os-Montes and Alta Douro (UTAD), Vila Real, Portugal; \\ 8BICSCOPE Group, LALVפREEUIMTE, Chemistry Department, Faculty of Science and Technology, NDVA University of Lisbon, Almada, Partugal; \\ PProteomass Scientific Saciety, Casta de Caparica, Portugal \\ *vanessasilva国utad.pt
}

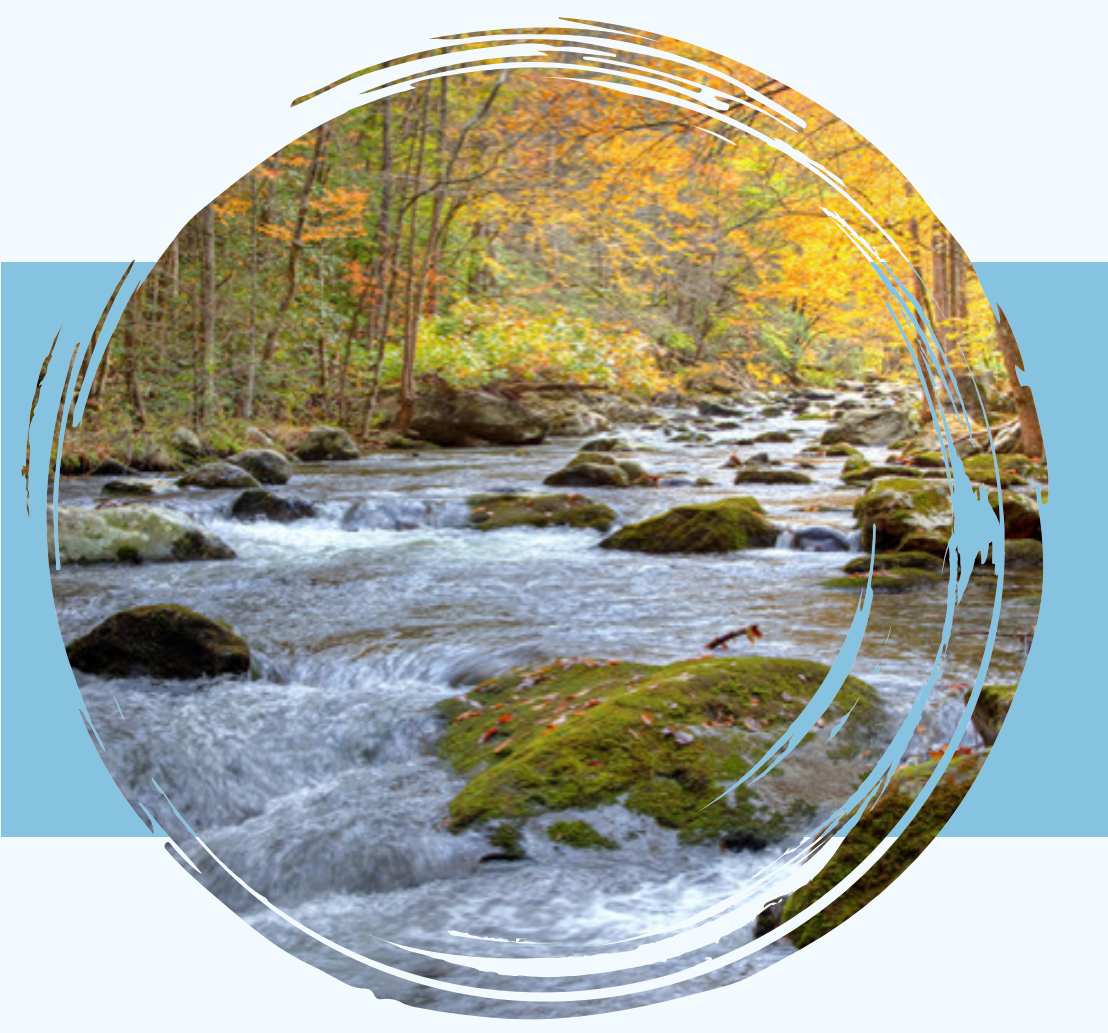

\section{Introduction}

Antimicrobial resistant bacteria in aquatic systems represents one the greatest challenges and emerging threats since water is one of the most relevant vehicles of bacterial dissemination. Monitoring the presence of antimicrobial resistance human pathogens, in particular staphylacacci, may lead to a better understanding of the epidemiology of infections caused by these bacteria. Thus, we aimed to investigate the prevalence of Staphylocaccus spp. in surface waters in Portugal.

\section{Methods}

Seventy-seven water samples (30 rivers, 18 streams, II irrigation systems, I0 dams, 5 fountains and 3 lakes) were collected fram the hydragraphic basins of Dauro river in Trás-as-Montes and Alto Douro region, Partugal. Samples were filtered through a cellulose nitrate pare membrane filter. The filters were incubated at 370 C far $24 \mathrm{~h}$ in BHl broth supplemented with 6.5\% of NaCl. After that, the inoculum was seeded on Mannital Salt agar plates and incubated at $370 \mathrm{C}$ far $24 \mathrm{~h}$. Staphylacacci species were identified by MALDI-TOF MS. Antimicrabial susceptibility testing was perfarmed by the Kirby-Bauer disk diffusion method.

\section{Results}

Seventy-five staphylococci were isolated from water samples of which 30 were coagulase-pasitive (CoPS) and 45 were coagulasenegative staphylocacci (CaNS). Among the CoPS, 29 were identified as $S$. aureus and one as $S$. pseudintermedius. The CoNS isolated included $S$. sciuri, $S$. lentus, $S$. xylosus and $S$. epidermidis, among others (Figure 1). S. aureus were susceptible to almost all antibiotics tested, and CaNS were mastly resistant to penicillin, clindamycin and fusidic acid.

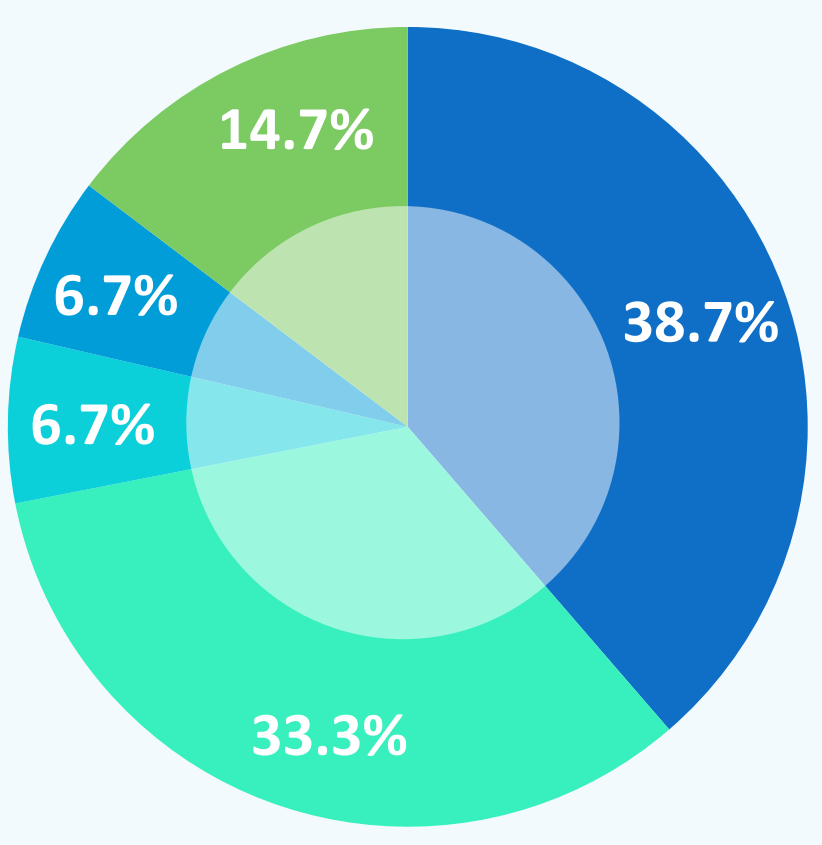

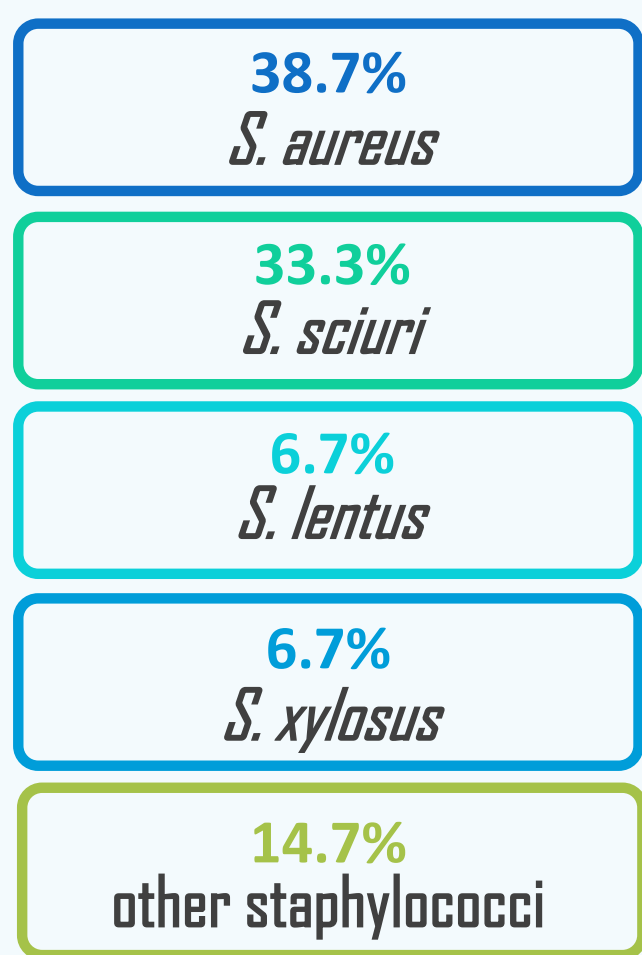

Figure I. Diversity of staphylacocci in surface water.

\section{Conclusions}

This study demonstrated that staphylocacci, including $S$. aureus, is present in surface waters. A high diversity of staphylocacci was detected in surface water samples, in particular for CoNS.

\section{Acknowledgements:}

This work was funded by the RED Project CAREBIDZ - Comparative assessment of antimicrobial resistance in environmental biofilms through proteomics - towards innovative theranostic biomarkers, with reference NDRTE-DI-DI45-FEDER-D3DIDI and PTDC/SAL-INF/30IDI/2017, financed by the European Regional Development Fund (ERDF) through the Northern Regional Dperational Program (NDRTE QO2Q) and the Foundation for Science and Technology (FCT). This work was also supported by the Assaciate Laboratory for Green Chemistry-LADV which is financed by national funds from FCT/MCTES (UID/QU//50006/2020). Vanessa Silva is grateful to FCT (Fundação para a Ciência e a Tecnolagia) for financial support through PhD grant SFRH/BD//37947/2018.

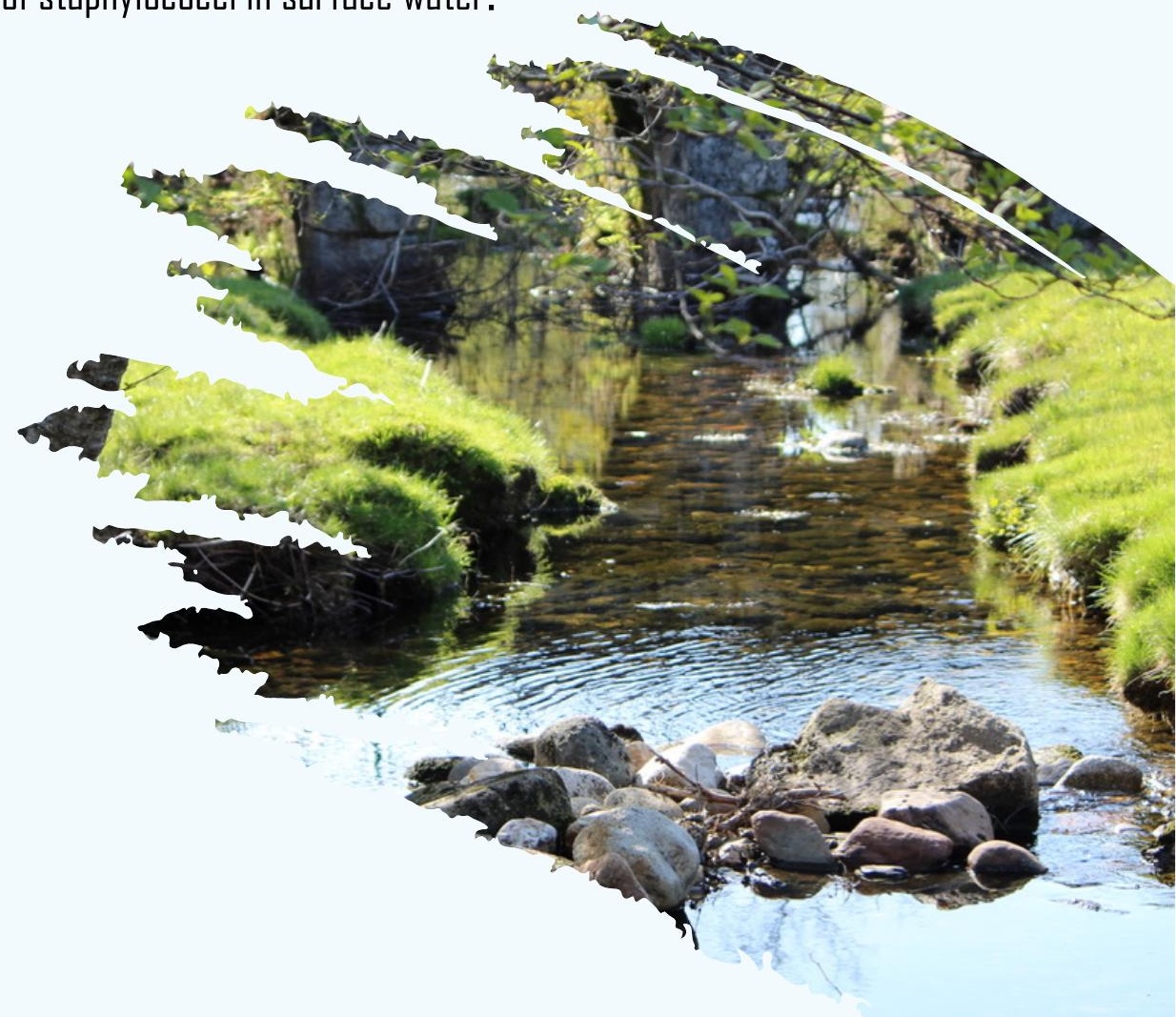

